\title{
Elemental abundance studies of CP stars
}

\author{
II. The silicon stars HD 133029 and HD $192913^{\star}$ \\ Z. López-García ${ }^{1, \star \star}$ and S.J. Adelman ${ }^{2}$ \\ 1 Observatorio Astronómico "Felix Aguilar", Universidad Nacional de San Juan, Argentina \\ 2 Department of Physics, The Citadel, 171 Moultrie Street, Charleston SC 29409, U.S.A.
}

Received November 2, 1998; accepted January 14, 1999

\begin{abstract}
Fine analyses of the silicon stars HD 133029 and HD 192913 are presented using ATLAS9 model atmospheres whose predictions fit the optical region spectrophotometry and $\mathrm{H} \gamma$ profiles and have the same bulk metallicity as the deduced abundances. Both are very $\mathrm{He}$ poor stars. The light elements are mostly solar except for silicon, and all the heavier elements, except nickel in HD 133029 which is solar, are greatly overabundant. The iron peak elements are typically 10 times overabundant. SrYZr are of order of 100 times solar. The rare earths are 1000 or more times overabundant.
\end{abstract}

Key words: stars: chemically peculiar - stars: abundances — stars: individual: HD 133029 — stars: individual: HD 192913

\section{Introduction}

This paper is the second of a series on the chemically peculiar (CP) stars, especially the magnetic types. We are systematically comparing their elemental abundances with those theories which try to explain their substantially nonsolar elemental abundances, such as the various radiative diffusion scenarios of Michaud (1970) and his collaborators. Paper I (Lopez-Garcia \& Adelman 1993) concerned the silicon star HD 43819 and the CP star HD 147550, which we discovered was a very cool $\mathrm{HgMn}$ star. For this paper we selected two silicon stars.

Bonsack (1977) studied the magnetic field and radial velocity of HD 133029 (= HR $5597=\mathrm{ADS}$

Send offprint requests to : S.J. Adelman

* Table 4 is is only available in electronic form at the CDS via anonymous ftp to cdsarc.u-strasbg.fr (130.79.128.5) or via http://cdsweb.u-strasbg.fr/Abstract.html

** Member of the Carrera del Investigador Científico, CONICET.
9477A) and found radial velocity variations with a period of 2.88706 days which were consistent with the photometric variations documented by Winzer (1974) and by Wolff \& Morrison (1975). Rather than periodic magnetic field strength variations, Bonsack found that significant and sometimes rapid ( $\geq 500 \mathrm{G}$ hour $^{-1}$ ) variations occur. His effective magnetic field strength measurements using Zeeman spectroscopy range from 850 to 3300 G. Borra \& Landstreet (1980) found +2905 to $4065 \mathrm{G}$ using $\mathrm{H} \beta$ and argued that Bonsack's observations are not necessarily incompatible with their observed smooth variability of $H_{\mathrm{e}}$. Bonsack (1977) found definite evidence that certain lines vary in strength and derived $v \sin i=21 \mathrm{~km} \mathrm{~s}^{-1}$, similar to Preston's (1971) upper limit. Spectrophotometry by Adelman \& White (1980) revealed quite broad continuum features at both $\lambda 4200$ and $\lambda 5200$ with some evidence for the $\lambda 6300$ feature. Recently Adelman (1998) reported that differential uvby photometry obtained with the Four College Automated Photometric Telescope (FCAPT) indicated a period of $2.88756 \pm 0.00004$ days and amplitudes of variability of 0.020 to 0.025 mag. Each magnitude has a single wave low amplitude light curve. These are crudely in phase, yet show details which suggest a complex photometric behavior whose explanation apparently requires regions with enhanced elemental abundances whose geometry is not that of spots at the magnetic poles; this is in accord with the magnetic diffusion hypothesis (Shore \& Adelman 1974, 1976) as the magnetic latitude at which maximum elemental abundance occurs does not necessarily have to be at the poles.

Osawa (1965) classified HD 192913 as a silicon star. It is one of the sharpest-lined Si stars with $v \sin i$ of $14 \mathrm{~km} \mathrm{~s}^{-1}$ (Preston 1971). Differential FCAPT uvby photometry of HD 192913 by Adelman \& Knox (1994) resulted in a period of $16.829 \pm 0.002$ days, a value which is close to previous studies by Bartolini et al. (1974), Winzer 
Table 1. $\mathrm{H} \gamma$ profiles

\begin{tabular}{ccc}
\hline$\Delta \lambda(\AA)$ & $R($ HD 133029) & $R($ HD 192913) \\
\hline 1 & 0.309 & 0.366 \\
2 & 0.385 & 0.459 \\
3 & 0.458 & 0.538 \\
4 & 0.530 & 0.606 \\
5 & 0.596 & 0.674 \\
6 & 0.654 & 0.732 \\
8 & 0.748 & 0.805 \\
10 & 0.809 & 0.853 \\
12 & 0.862 & 0.893 \\
14 & 0.892 & 0.914 \\
16 & 0.917 & 0.929 \\
18 & 0.935 & 0.946 \\
20 & 0.942 & 0.952 \\
22 & 0.952 & 0.959 \\
24 & 0.960 & 0.964 \\
28 & 0.968 & 0.976 \\
32 & 0.974 & 0.985 \\
36 & 0.978 & 0.994 \\
40 & 0.983 & 0.999 \\
\hline
\end{tabular}

(1974), Schoneich et al. (1976), and Musielok et al. (1980). This star shows an asymmetric single wave photometric variability with the broad minimum showing evidence for two sub-minima. Optical spectrophotometry (Adelman 1982) showed a definite $\lambda 5200$ feature while the other three spectrophotometric anomalies are absent (Adelman \& Pyper 1993). Ryabchikova et al. (1990) concluded that the metal lines change with the photometric period.

\section{Observational material and line identifications}

Four nitrogen-baked IIa-O $4.3 \AA \mathrm{mm}^{-1}$ spectrograms (Ce 22760, Ce 22922, Ce 23322 and Ce 23760) of HD 133029 and four (Ce 21996, Ce 22005, Ce 22015 and Ce 22557) of HD 192913, obtained by SJA with the coudé spectrograph of the $2.5 \mathrm{~m}$ telescope of Mount Wilson Observatory were used in this study. The region covered is approximately $\lambda \lambda 3760-4650$.

There are no published line identifications of HD 133029. The stellar lines were identified with the aid of standard sources, especially, A Multiplet Table of Astrophysical Interest (Moore 1945). Lines of H I, He I, C II, Mg I, Mg II, Al I, Si II, S II, Ca II, Ti II, Cr I, Cr II, Mn II, Fe I, Fe II, Fe III, Ni II, Y II, Sr II, Zr II, Ce II, Nd II, and Eu II are definitely present. Those of Si III and $\mathrm{Hg}$ II are probable identifications.

Jaschek \& López-García's (1966) detailed identification study of HD 192913 for $\lambda \lambda 3754-4670$ based on a $4.5 \AA \mathrm{mm}^{-1}$ Palomar Observatory spectrogram listed lines of H I, C II, Mg II, Al I, Si II, Ca II, Sc II, Ti II, V II, Cr I, Cr II, Mn II, Fe I, Fe II, Fe III, Sr II, Y II, Zr II, Eu II, Gd II, Hg I, and Hg II. Cowley \& Crosswite (1978) (see also Ryabchikova et al. 1990) identified lines of several intermediate and heavy singly and doubly ionized rareearth species, in particular Ce III, Nd III, Sm III, Gd III, Dy III, Ho III, Er III, Tm III, and Yb III.

Equivalent widths and $\mathrm{H} \gamma$ profiles were read directly from the intensity tracings which were made with the PDS microdensitometer of Kitt Peak National Observatory. Those from different spectrograms of the same star were averaged to increase the signal-to-noise ratio, but this means averaging over any spectral variability. As the $\mathrm{H} \gamma$ profiles were assumed to be symmetric about the line core, their wings were averaged.

\section{Atmospheric parameters}

Temperature estimates for HD 133029 were made by Burbidge \& Burbidge (1955), Megessier (1971, 1988), and Adelman \& White (1980) and for HD 192913 by Adelman (1982) and Megessier (1988). To improve these values, we used the uvby $\beta$ mean colors of Hauck \& Mermilliod (1980) with the calibration of Napiwotzki et al. (1993) and obtained $T_{\text {eff }}=11760 \mathrm{~K}, \log g=4.10$ for HD 133029 and $T_{\text {eff }}=11760 \mathrm{~K}, \log g=3.30$ for HD 192913. We calculated line blanketed ATLAS9 model atmospheres (Kurucz 1995) with 10 times solar metallicity, and compared their predicted fluxes with dereddened spectrophotometry and the observed $\mathrm{H} \gamma$ profiles with synthetic spectra of the $\mathrm{H} \gamma$ region calculated with SYNTHE (Kurucz \& Avrett 1981) (Table 1). For HD 133029 we found $T_{\text {eff }}=11200 \pm 100 \mathrm{~K}$, $\log g=3.84 \pm 0.10$ dex. As discussed by Adelman et al. (1995), these model predictions fit the energy distribution reasonably well including the $\lambda 5200$ broad continuum region. For HD 192913 we found in this manner, $T_{\text {eff }}=10900 \pm 100 \mathrm{~K}, \log g=3.40 \pm 0.10$ dex.

\section{Abundance analyses}

We determined the metal abundances from the equivalent widths with Program WIDTH9 (Kurucz 1995). The adopted metal line damping constants were the default semi-classical approximations except for those of neutral and singly-ionized $\mathrm{Ca}-\mathrm{Ni}$ lines whose values are based on the data of Kurucz (1995), for lines of C II multiplet 6 and Mg II multiplet 4 where the adopted values for the Stark broadening were based on data of Sahal-Brechot (1969) and for Si II and Ca II where the damping constants are those of Lanz et al. (1988) and Chapelle \& Sahal-Brechot (1970) respectively.

We calculated abundances from Fe I and Fe II lines for a range of possible microturbulent velocities $(\xi)$. For the final values, (Table 2), the abundances are not 
Table 2. Determination of microturbulent velocity assuming no magnetic field

\begin{tabular}{cccccccc}
\hline Star & Species & $n$ & $\begin{array}{c}\left(\xi_{1}\right) \\
\left(\mathrm{km} \mathrm{s}^{-1}\right)\end{array}$ & $\log \mathrm{Fe} / N_{\mathrm{T}}$ & $\begin{array}{c}\left(\xi_{2}\right) \\
\left(\mathrm{km} \mathrm{s}^{-1}\right)\end{array}$ & $\log \mathrm{Fe} / N_{\mathrm{T}}$ & $g f$-values \\
\hline HD 133029 & Fe I & 39 & 2.4 & $-3.32 \pm 0.22$ & 2.2 & $-3.29 \pm 0.22$ & MF\&KX \\
& & 36 & 2.4 & $-3.33 \pm 0.22$ & 2.5 & $-3.35 \pm 0.22$ & MF \\
& Fe II & 72 & 2.0 & $-3.20 \pm 0.26$ & 2.2 & $-3.24 \pm 0.26$ & MF\&KX \\
& & 21 & 2.8 & $-3.53 \pm 0.24$ & 2.6 & $-3.46 \pm 0.24$ & MF \\
& mean $\xi:$ & $2.4 \mathrm{~km} \mathrm{~s}^{-1}$ & & & & & \\
& Ti II & 31 & 1.7 & $-5.93 \pm 0.26$ & 1.6 & $-5.92 \pm 0.26$ & MF\&KX \\
& Cr II & 46 & 2.9 & $-4.29 \pm 0.31$ & 2.8 & $-4.26 \pm 0.31$ & MF\&KX \\
HD 192913 & Fe I & 51 & 0.7 & $-3.23 \pm 0.19$ & 0.8 & $-3.24 \pm 0.19$ & MF\&KX \\
& & 50 & 0.7 & $-3.23 \pm 0.19$ & 0.8 & $-3.25 \pm 0.19$ & MF \\
& Fe II & 71 & 1.0 & $-3.39 \pm 0.20$ & 1.0 & $-3.39 \pm 0.20$ & MF\&KX \\
& & 32 & 1.0 & $-3.40 \pm 0.20$ & 1.0 & $-3.40 \pm 0.20$ & MF \\
& mean $\xi:$ & $0.9 \mathrm{~km} \mathrm{~s}^{-1}$ & & & & & \\
& Ti II & 44 & 1.2 & $-5.66 \pm 0.25$ & 1.2 & $-5.66 \pm 0.25$ & MF\&KX \\
& Cr II & 37 & 1.2 & $-4.97 \pm 0.20$ & 1.1 & $-4.96 \pm 0.20$ & MF\&KX \\
\hline
\end{tabular}

Table 3. Magnetic field determinations

\begin{tabular}{cccccccc}
\hline \multirow{2}{*}{ Star } & Species & $n$ & $\begin{array}{c}H_{1} \\
(\mathrm{kG})\end{array}$ & $\log N / N_{\mathrm{T}}$ & $\begin{array}{c}H_{2} \\
(\mathrm{kG})\end{array}$ & $\log N / N_{\mathrm{T}}$ & $g f$-values \\
\hline HD 133029 & Fe I & 39 & 3.1 & $-3.29 \pm 0.21$ & 3.3 & $-3.32 \pm 0.22$ & MF\&KX \\
& & 36 & 3.1 & $-3.31 \pm 0.22$ & 3.1 & $-3.31 \pm 0.22$ & MF \\
& Fe II & 72 & 2.8 & $-3.18 \pm 0.30$ & 3.0 & $-3.21 \pm 0.30$ & MF\&KX \\
& & 21 & 3.5 & $-3.37 \pm 0.37$ & 3.5 & $-3.37 \pm 0.37$ & MF \\
& mean H: & $3.2 \mathrm{kG}$ & & & & & \\
& Ti II & 30 & 3.0 & $-5.98 \pm 0.26$ & 3.1 & $-5.99 \pm 0.26$ & MF\&KX \\
& Cr II & 23 & 4.9 & $-4.33 \pm 0.30$ & 4.9 & $-4.35 \pm 0.31$ & MF\&KX \\
HD 192913 & & & & & & & \\
& Fe I & 51 & 1.0 & $-3.24 \pm 0.19$ & 1.0 & $-3.24 \pm 0.19$ & MF\&KX \\
& & 50 & 1.0 & $-3.24 \pm 0.19$ & 1.1 & $-3.25 \pm 0.19$ & MF \\
& Fe II & 71 & 1.4 & $-3.40 \pm 0.23$ & 1.4 & $-3.40 \pm 0.23$ & MF\&KX \\
& & 32 & 1.8 & $-3.54 \pm 0.26$ & 1.7 & $-3.51 \pm 0.25$ & MF \\
& mean H: & $1.3 \mathrm{kG}$ & & & & & \\
& Ti II & 44 & 2.3 & $-5.74 \pm 0.24$ & 2.2 & $-5.72 \pm 0.23$ & MF\&KX \\
& Cr II & 37 & 1.8 & $-4.96 \pm 0.20$ & 1.7 & $-4.95 \pm 0.20$ & MF\&KX \\
\hline
\end{tabular}

dependent on the equivalent widths $\left(\xi_{1}\right)$ or minimize the rms scatter of the abundances $\left(\xi_{2}\right)$. Values for both species were derived using lines with $g f$ values only from Martin et al. (1988) and also with $g f$-values from compatible sources, in this case Kurucz (1995). From these species a mean microturbulence of $2.4 \mathrm{~km} \mathrm{~s}^{-1}$ is found for HD 133029. The Cr II lines give a value of $2.9 \mathrm{~km} \mathrm{~s}^{-1}$ while the Ti II lines suggest $1.7 \mathrm{~km} \mathrm{~s}^{-1}$. For HD 192913, the Fe I and Fe II lines indicate a mean microturbulence of $0.9 \mathrm{~km} \mathrm{~s}^{-1}$ while Ti II and Cr II lines $1.2 \mathrm{~km} \mathrm{~s}^{-1}$. No microturbulence is expected if the magnetic $\mathrm{CP}$ stars have quiescent atmospheres as required by various radiative diffusion scenarios (see, e.g. Michaud 1970). The derived microturbulence is most likely a manifestation of an organized weak magnetic field with each line having its own effective microturbulence due to the width and the distribution of its Zeeman components. Strong lines with wide patterns and many components will be desaturated more easily than strong lines with smaller patterns and fewer components. Following Adelman (1973) we equated the mean width of the Zeeman $\sigma$ components to the Doppler broadening to derive an effective microturbulence for each line which increases with the strength of the magnetic field and the width of the Zeeman pattern. Although this approximation is adequate for the current data, more refined modeling will be required for observations with somewhat greater signal-to-noise ratios.

By assuming that there is no microturbulence and requiring that the abundances be independent of the derived magnetic field $\left(H_{1}\right)$ or that the scatter in the derived abundances be a minimum $\left(H_{2}\right)$ we found that surface magnetic field of HD 133029 (Table 3 ) is about $3.2 \mathrm{kG}$ from the Fe I and Fe II lines. The Cr II lines yield $4.9 \mathrm{kG}$ 
Table 5. Sensitivity of the derived abundances of HD 192913 to changes in temperature and surface gravity

\begin{tabular}{lccc}
\hline Elements & $\begin{array}{c}\text { Log } N / H \text { for } \\
\text { adopted model }\end{array}$ & $\begin{array}{c}\text { Log } N / H \text { for } 500 \mathrm{~K} \\
\text { hotter model }\end{array}$ & $\begin{array}{c}\text { Log } N / H \text { for } 0.5 \\
\text { dex greater model }\end{array}$ \\
\hline C II & -3.16 & -3.08 & -2.96 \\
Mg I & -5.10 & -4.88 & -5.32 \\
Mg II & -4.70 & -4.66 & -4.70 \\
Al I & -5.33 & -5.20 & -5.44 \\
Si II & -4.06 & -3.99 & -4.11 \\
S II & -4.67 & -4.61 & -4.46 \\
Ca II & -5.33 & -5.15 & -5.47 \\
Sc II & -8.68 & -8.52 & -8.72 \\
Ti II & -5.60 & -5.42 & -5.60 \\
Cr I & -3.60 & -3.27 & -3.78 \\
Cr II & -4.92 & -4.86 & -4.82 \\
Mn II & -4.76 & -4.71 & -4.68 \\
Fe I & -3.26 & -3.06 & -3.41 \\
Fe II & -3.36 & -3.32 & -3.24 \\
Fe III & $-2.68:$ & $-2.63:$ & $-2.40:$ \\
Ni II & -5.26 & -5.29 & -5.10 \\
Sr II & -5.03 & -4.84 & -5.21 \\
Y II & -7.59 & -7.40 & -7.67 \\
Zr II & -7.22 & -7.04 & -7.25 \\
Ba II & -8.52 & -8.35 & -8.61 \\
Ce II & -6.86 & -6.69 & -6.94 \\
Pr II & -6.66 & -6.47 & -6.76 \\
Nd II & -6.81 & -6.64 & -6.90 \\
Sm II & -6.34 & -6.14 & -6.43 \\
Eu II & -5.29 & -5.10 & -5.45 \\
Gd II & -7.44 & -7.29 & -6.52 \\
Dy II & -6.56 & -6.33 & -5.00 \\
Hg II & -5.12 & -5.10 & \\
\hline
\end{tabular}

and the Ti II lines $3.1 \mathrm{kG}$. For HD 192913 we found a surface magnetic field of about $1.3 \mathrm{kG}$ from the Fe I and Fe II lines, while $\mathrm{Ti}$ II lines indicated $2.30 \mathrm{kG}$ and $\mathrm{Cr}$ II lines $1.8 \mathrm{kG}$. As the rms values about the mean are similar for both methods, we present the final abundance results for the assumption of a uniform microturbulence of $2.4 \mathrm{~km} \mathrm{~s}^{-1}$ for HD 133029 and $0.9 \mathrm{~km} \mathrm{~s}^{-1}$ for HD 192913.

We calculate the He I profiles in LTE from the model atmospheres with the program SYNSPEC (Hubeny et al. 1994). Only He I $\lambda 4026$ is present for HD 133029 and its equivalent width is about $13 \mathrm{m \AA}$. Thus HD 133029 is very He poor. For HD 192913 no He I lines were seen, making it too a very He poor star.

In Table 4 we present the analyses of the line spectra. For each line we give the multiplet number (Moore 1945), the laboratory wavelength, the $g f$ value and its source, the equivalent width in $\mathrm{m} \AA$, and the derived abundance $\log N / N_{\mathrm{T}}$. We did not include seriously blended lines in the analyses. To give an idea of the sensitivity of our results to errors in effective temperature and surface gravity, we derived the abundances of HD 192913 also for models $500 \mathrm{~K}$ hotter and $\log g 0.5$ dex greater. Table 5 shows the size of the resultant changes.

\section{Discussion}

Table 6 compares the derived abundances for HD 133029 and HD 192913 with solar values (Grevesse et al. 1996). Also given are the abundances of HD 43819 (Lopez-Garcia \& Adelman 1994). Compared with the Sun, the light elements of the silicon stars are mostly solar except for $\mathrm{Si}$, and all the heavier elements except nickel in HD 133029, which is solar, are greatly overabundant. HD 133029 has underabundant C, Mg, and S and overabundant Ca while HD 192913 underabundant Mg. The iron peak elements are typically 10 times overabundant except $\mathrm{Cr}$ in HD 133029 which is 100 times solar, Mn in HD 192913 which is of order 100 times solar, and Ni which is highly variable. SrYZr are of order 100 times solar when observed. The rare earths are 1000 or more times overabundant. HD 43819 appears to be in general a slightly less metal rich star than the other two silicon stars.

For a substantial confrontation with theory, we need to analyze additional magnetic CP stars with higher signalto-noise spectra. This will also give additional information on the line-to-line magnetic intensification. We need to extend the range of effective temperatures to look for temperature dependences and for theoretical tests. 
Table 6. Comparison of derived and solar abundances

\begin{tabular}{lcccr}
\hline Elements & $\begin{array}{c}\text { HD } 192913 \\
\log N / H\end{array}$ & $\begin{array}{c}\text { HD } 133029 \\
\log N / H\end{array}$ & $\begin{array}{c}\text { HD } 43819 \\
\log N / H\end{array}$ & $\begin{array}{r}\text { Sun } \\
\log N H\end{array}$ \\
\hline C II & -3.16 & -3.08 & -3.78 & -3.45 \\
Mg I & -5.10 & $\ldots$ & -5.01 & -4.42 \\
Mg II & -4.70 & -4.26 & -4.72 & -4.42 \\
Al I & -5.33 & $\ldots$ & $\ldots$ & -5.53 \\
Si II & -4.06 & -3.36 & -3.88 & -4.45 \\
S II & -4.67 & -4.84 & -5.46 & -4.67 \\
Ca II & -5.33 & -5.72 & -5.12 & -5.64 \\
Sc II & -8.68 & $\ldots$ & -9.11 & -8.83 \\
Ti II & -5.60 & -6.02 & -5.81 & -6.98 \\
Cr I & -3.60 & $\ldots$ & -4.69 & -6.33 \\
Cr II & -4.92 & -4.15 & -5.02 & -6.33 \\
Mn II & -4.76 & -5.00 & $-5.55:$ & -6.61 \\
Fe I & -3.26 & -3.32 & -3.48 & -4.50 \\
Fe II & -3.36 & -3.28 & -3.66 & -4.50 \\
Fe III & $-2.68:$ & -3.18 & -3.70 & -4.50 \\
Ni II & -5.26 & -5.70 & -6.49 & -5.75 \\
Sr II & -5.03 & -7.01 & -5.63 & -9.03 \\
Y II & -7.59 & -7.79 & $\ldots$ & -9.76 \\
Zr II & -7.22 & -7.53 & -7.81 & -9.40 \\
Ba II & -8.52 & -8.68 & $\ldots$ & -9.87 \\
Ce II & -6.86 & -7.14 & -6.86 & -10.42 \\
Pr II & -6.66 & -6.98 & -6.69 & -11.29 \\
Nd II & -6.81 & -6.72 & -7.34 & -10.50 \\
Sm II & -6.34 & $\ldots$ & $\ldots$ & -10.99 \\
Eu II & -5.29 & -8.44 & -8.15 & -11.49 \\
Gd II & -7.44 & $\ldots$ & $-7.18:$ & -10.88 \\
Dy II & -6.56 & $\ldots$ & $\ldots$ & -10.86 \\
Hg II & -5.12 & -6.57 & $\ldots$ & -10.83 \\
$T_{\text {eff }}$ & 10900 & 11200 & 11300 & \\
$\log g$ & 3.40 & 3.84 & 3.20 & \\
\hline & & & & \\
\hline
\end{tabular}

Acknowledgements. ZLG thanks the Director of CASLEO for the use of their facilities in connection with this project. Her research was support by a grant from the CICYTCA, UNSJ. SJA acknowledges partial support from grants from The Citadel Development Foundation. Both authors appreciate Drs. R.L. Kurucz and I. Hubeny for making their codes available to them, and Mrs. C. Puigdengolas for partial measurement of the equivalent widths.

\section{References}

Adelman S.J., 1973, ApJ 183, 95

Adelman S.J., 1982, A\&AS 49, 663

Adelman S.J., 1998, A\&AS 128, 245

Adelman S.J., Knox J.R., 1994, A\&AS 103, 1

Adelman S.J., Pyper D.M., 1993, A\&AS 101, 393

Adelman S.J., Pyper D.M., López-García Z., Caliskan H., 1995, A\&A 296, 467

Adelman S.J., White R.E., 1980, A\&AS 42, 289

Bartolini C., et al., 1974, IBVS, 48

Biemont E., Grevesse N., Hannaford P., Lowe R.M., 1981, ApJ 248,867

Biemont E., Karner C., Meyer G., Trager F., zu Putlitz G., 1982, A\&A 107, 166
Bonsack W.K., 1977, A\&A 59, 195

Borra E.F., Landstreet J.D., 1980, ApJS 42, 421

Burbidge E.M., Burbidge G.R., 1955, ApJ 122, 396

Chapelle J., Sahal-Brechot S., 1970, A\&A 6, 415

Cowley C.R., Crosswhite H.M., 1978, PASP 90, 108

Dworetsky M.M., 1980, PASP 84, 652

Fuhr J.R., Martin G.A., Wiese W.L., 1988, J. Phys. Chem. Ref. Data 17, Suppl. 4

Grevesse N., et al., 1981, Upper Main Sequence CP Stars, 23rd. Liège Astrophys. Colloq., Upper Main Sequence CP Stars, Institut d'Astrophysique, Université de Liège, p. 211

Grevesse N., Noels A., Sauval A.J., 1996, in Holt S.S. \& Sonneborn G. (eds.) ASP Conf. Ser. 99, Cosmic Abundances, ASP, San Francisco, p. 117

Hannaford P., Lowe R.M., Grevesse N., Biemonet E., 1982, ApJ 261, 736

Hauck B., Mermilliod M., 1980, A\&AS 40, 1

Hubeny I., Lanz T., Jeffery C.S., 1994, Daresbury Laboratory Newsletter on Analyses of Astronomical Spectra Nos. 20, 30

Jaschek M., López García Z., 1966, ZfA 64, 217

Kurucz R.L., 1979, ApJS 40, 1

Kurucz R.L., Avrett E.H., 1981, SAO Special Report No. 391 Kurucz R.L., 1995, in: Adelman S.J., Kupka F. \& Weiss W.W. (eds.) ASP Conf. Ser. 108, Model Atmospheres and Spectrum Synthesis, ASP, San Francisco, p. 205 
Lanz T., Artru M.C., 1985, Phys. Scr. 32, 115

Lanz T., et al., 1988, A\&A 192, 249

López-García Z., Adelman S.J., 1994, A\&AS 107, 353

Magazzu A., Cowley C.R., 1986, ApJ 308, 254

Martin G.A., Fuhr J.R., Wiese W.L., 1988, J. Phys. Chem. Ref. Data 15, Suppl. 3

Megessier C., 1971, A\&A 10, 332

Megessier C., 1988, A\&AS 72, 551

Michaud G., 1970, ApJ 160, 641

Moore C.E., 1945, A Multiplet Table of Astrophysical Interest. Princeton University Observatory, Princeton

Musielok B., et al., 1980, Astron. Nachr. 301, 71

Napiwotzki R., Schonberner D., Wenske V., 1993, A\&A 268, 653

Osawa K., 1965, Ann. Tokio Astron. Obs., Second Series 9, p. 123

Preston G.W., 1971, ApJ 164, 309
Ryabchikova T.A., Davidova E.S., Adelman S.J., 1990, PASP 102,581

Sahal-Brechot S., 1969, A\&A 2, 322

Schoneich W., et al., 1976, Astron. Nachr. 297, 173

Shore S.N., Adelman S.J., 1974, ApJ 191, 165

Shore S.N., Adelman S.J., 1976, in Physics of Ap Stars, IAU Coll. 32 , p. 125

Ward L., 1985, MNRAS 213, 71

Wiese W.L., Martin G.A., 1980, NSRDS-NBS 68, Part 2, US Gov. Printing Office, Washington DC

Wiese W.L., Smith M.W., Glennon B.M., 1966, NSRDS-NBS 4, US Gov. Printing Office, Washington DC

Wiese W.L., Smith M.W., Miles B.M., 1969, NSRDS-NBS 22, US Gov. Printing Office, Washington DC

Winzer J.E., 1974, Ph.D. Thesis, University of Toronto

Wolff S.C., Morrison N.D., 1975, PASP 87, 231 